\title{
Avoider-Forcer games on hypergraphs with small rank
}

\author{
Małgorzata Bednarska-Bzdȩga \\ Department of Discrete Mathematics \\ Adam Mickiewicz University \\ Poznań, Poland \\ mbed@amu.edu.pl
}

Submitted: Jan 25, 2013; Accepted: Dec 29, 2013; Published: Jan 12, 2014

Mathematics Subject Classifications: 91A46, 91A43

\begin{abstract}
We study biased $(a: f)$ Avoider-Forcer games played on hypergraphs, in the strict and monotone versions. We give a sufficient condition for Avoider's win, useful in the case of games on hypergraphs whose rank is smaller than $f$. We apply this result to estimate the threshold bias in Avoider-Forcer $(1: f)$ games in which Avoider is trying not to build a copy of a fixed graph $G$ in $K_{n}$. We also study the $d$-degree $(1: f)$ game in which Avoider's aim is to avoid a spanning subgraph of minimal degree at least $d$ in $K_{n}$. We show that the strict 1-degree game has the threshold which is the same as the threshold of the Avoider-Forcer connectivity game.
\end{abstract}

Keywords: combinatorial games, Avoider-Forcer games, Avoider-Enforcer games

\section{Introduction}

Let $V$ be a finite set and $E$ be a multiset of subsets of $V$. We consider two types of two-person positional games played on the hypergraph $\mathcal{H}=(V, E)$; these are AvoiderForcer strict games (cf. [3] and [10]) and Avoider-Forcer games in the monotone version (introduced in [9]). We will relate these games with the widely studied and well understood Maker-Breaker games.

Let us recall that in Maker-Breaker $(p: q)$ games, where $p$ and $q$ are natural numbers, two players claim in turns previously unselected (free) elements of $V$ until all elements of $V$ are selected. In every turn Maker selects $p$ vertices and Breaker answers by selecting $q$ vertices of the hypergraph $\mathcal{H}$. Maker wins if and only if by the end of the game he occupies all elements of at least one edge of $\mathcal{H}$. We will denote such games by $\mathcal{M B}(\mathcal{H}, p, q)$. 
In Avoider-Forcer strict $(a: f)$ game, denoted by $\mathcal{A F}(\mathcal{H}, a, f)$, in each turn Avoider selects exactly $a$ elements and then Forcer selects exactly $f$ elements of $V$. If in the last turn there are fewer free vertices than a player has to select, he takes all the remaining unoccupied elements. Avoider loses if by the end of the game he selects all elements in at least one edge of the hypergraph, otherwise he wins.

The rules of Avoider-Forcer $(a: f)$ games in the monotone version are almost the same as those of $\mathcal{A F}(\mathcal{H}, a, f)$. The only difference is that Avoider and Forcer select at least $a$ and at least $f$ elements per turn respectively. We denote such games by $\mathcal{A F}_{\text {mon }}(\mathcal{H}, a, f)$.

Let us remark that we follow the game terminology of [3], while the authors of [6], [8], [9], [10] and [12] call Forcer Enforcer. We also emphasize that by the $i$ th turn we mean the pair of Avoider's $i$ th move and Forcer's $i$ th move.

An important tool used in studying Avoider-Forcer games is the following winning condition for Avoider, proved by Hefetz, Krivelevich and Szabó. The condition was proved in [10] for $\mathcal{A F}(\mathcal{H}, a, f)$, and the authors of [9] noted that it applies also to $\mathcal{A F}_{\text {mon }}(\mathcal{H}, a, f)$.

Theorem 1.1 ([10], Thm 1.1). If

$$
\sum_{D \in E(\mathcal{H})}\left(\frac{1}{a}+1\right)^{-|D|+a}<1
$$

then for every $f \geqslant 1$, Avoider has a winning strategy for the games $\mathcal{A F}(\mathcal{H}, a, f)$ and $\mathcal{A F}_{\text {mon }}(\mathcal{H}, a, f)$.

If

$$
\sum_{D \in E(\mathcal{H})}\left(\frac{1}{a}+1\right)^{-|D|}<1
$$

$f \geqslant 1$ and Forcer makes the last move in $\mathcal{A F}(\mathcal{H}, a, f)$, then Avoider has a winning strategy for $\mathcal{A} \mathcal{F}(\mathcal{H}, a, f)$.

Let us define the rank of a hypergraph by setting

$$
\operatorname{rank}(\mathcal{H})=\max _{D \in E(\mathcal{H})}|D| .
$$

In this paper we give the following Avoider's winning condition, which is useful in games played on hypergraphs of small rank.

Theorem 1.2. Let $\mathcal{H}$ be a hypergraph of rank $r$.

(i) If

$$
\sum_{D \in E(\mathcal{H})}\left(\frac{f}{a r}+1\right)^{-|D|+a}<1
$$

then Avoider has a winning strategy for both $\mathcal{A F}(\mathcal{H}, a, f)$ and $\mathcal{A} \mathcal{F}_{\text {mon }}(\mathcal{H}, a, f)$. 
(ii) Let

$$
\sum_{D \in E(\mathcal{H})}\left(\frac{f}{a r}+1\right)^{-|D|}<1 .
$$

Then for every $f^{\prime} \geqslant f$, in the course of $\mathcal{A F}\left(\mathcal{H}, a, f^{\prime}\right)$ and $\mathcal{A} \mathcal{F}_{\text {mon }}\left(\mathcal{H}, a, f^{\prime}\right)$, in each position with at least $a+f$ free vertices, Avoider has a move after which no edge of $\mathcal{H}$ is entirely occupied by him.

The first inequality in the above theorem, for $a=1$, looks similar to the well known Beck's winning condition for Breaker in $\mathcal{M B}(\mathcal{H}, 1, f)$ (cf. [2]):

$$
\sum_{D \in E(\mathcal{H})}(f+1)^{-|D|+1}<1 .
$$

However, we cannot expect that (1) is in general a sufficient condition for Avoider's win in $\mathcal{A F}_{\text {mon }}(\mathcal{H}, 1, f)$. Furthermore, for any $a \geqslant 1$ we cannot improve the expression $\left(\frac{f}{a r}+1\right)$ in Theorem 1.2(i) more than up to $\left(\frac{3 f}{a r}+1\right)$. We express this fact by Theorem 1.3:

Theorem 1.3. Let $a \geqslant 1$ and $r \geqslant 3 a$. There exists a constant $c$ (depending on $r$ and a) such that for every $f>c$ there exists a hypergraph $\mathcal{H}$ of rank $r$ which satisfies the condition

$$
\sum_{D \in E(\mathcal{H})}\left(\frac{3 f}{a r}+1\right)^{-|D|+a}<1,
$$

but Forcer has a winning strategy for $\mathcal{A F}_{\text {mon }}(\mathcal{H}, a, f)$.

The construction of a hypergraph described in the above theorem is very simple. We will consider edge-disjoint, $r$-uniform hypergraphs and apply the results on Box games by Ferber, Krivelevich and Naor [6].

In order to demonstrate how to use Theorem 1.2, we study the threshold properties of Avoider-Forcer $(1: f)$ games. As observed in [9] and [10], for such games one can consider three types of thresholds. Thus, given a hypergraph $\mathcal{H}$, we denote by the lower threshold bias $f_{\mathcal{H}}^{-}$the largest integer such that for every $f \leqslant f_{\mathcal{H}}^{-}$Forcer has a winning strategy for $\mathcal{A} \mathcal{F}(\mathcal{H}, 1, f)$. We mean by the upper threshold bias $f_{\mathcal{H}}^{+}$the largest integer $f$ such that Forcer wins $\mathcal{A F}(\mathcal{H}, 1, f)$. Both $f_{\mathcal{H}}^{-}$and $f_{\mathcal{H}}^{+}$always exist, except for some degenerate cases. If $f_{\mathcal{H}}^{+}=f_{\mathcal{H}}^{-}$, then we call the number $f_{\mathcal{H}}=f_{\mathcal{H}}^{+}=f_{\mathcal{H}}^{-}$the threshold bias of the strict game. Finally, the threshold bias of the monotone game is the non-negative integer $f_{\mathcal{H}}^{\text {mon }}$ such that Forcer wins $\mathcal{A F}_{\text {mon }}(\mathcal{H}, 1, f)$ iff $f \leqslant f_{\mathcal{H}}^{\text {mon }}$. Throughout the paper the (upper/lower/monotone) threshold bias is called shortly the (upper/lower/monotone) threshold. Note that the monotonicity rules of $\mathcal{A F}_{\text {mon }}(\mathcal{H}, 1, f)$ guarantee the existence of $f_{\mathcal{H}}^{\text {mon }}$, as opposed to the strict Avoider-Forcer games, in which the threshold $f_{\mathcal{H}}$ may not exist (cf. [10]).

The threshold properties of Avoider-Forcer games were studied extensively for graph games. In such games the players select edges of the complete graph $K_{n}$, Avoider's aim is to avoid a graph from a given family of subgraphs of $K_{n}$ (see e.g. [8],[9],[10],[12]). Let us 
mention some examples of graph games, studied before in both strict and monotone versions. In connectivity games Avoider is trying to avoid any connected spanning subgraph of $K_{n}$. In other words, the connectivity games $\mathcal{A F}\left(\mathcal{C}_{1, n}, 1, f\right)$ and $\mathcal{A F}_{\text {mon }}\left(\mathcal{C}_{1, n}, 1, f\right)$ are played on the hypergraph $\mathcal{C}_{1, n}$, with vertex-set $V\left(\mathcal{C}_{1, n}\right)=E\left(K_{n}\right)$, whose edges are connected spanning subgraphs of $K_{n}$. In a similar way, the hypergraphs $\mathcal{C}_{d, n}, \mathcal{C}_{d, n}^{\prime}, \mathcal{H a m}_{n}$ and $\mathcal{M}_{n}$ correspond to the families of all $d$-connected spanning subgraphs, $d$-edge-connected spanning subgraphs, Hamiltonian cycles and perfect matchings in $K_{n}$ respectively. To avoid technicalities we always assume that $n$ is even when considering $\mathcal{M}_{n}$. We denote by $\mathcal{D}_{d, n}$, with $1 \leqslant d \leqslant n-1$, the hypergraph corresponding to the family of all spanning subgraphs of the minimum degree at least $d$. Finally, given a graph $G$, by $\mathcal{H}_{G, n}$ we denote the hypergraph corresponding to the family of all copies of $G$ in $K_{n}$. We refer to games played on the hypergraphs $\mathcal{H a m}_{n}, \mathcal{C}_{d, n}, \mathcal{D}_{d, n}$ and $\mathcal{H}_{G, n}$, as Hamiltonicity, d-connectivity, $d$-degree and small-graph games respectively.

Somewhat surprisingly the thresholds for monotone Avoider-Forcer Hamiltonicity, $d$-connectivity and $d$-degree games (with $d$ fixed) are very similar to their Maker-Breaker counterparts. Krivelevich and Szabó [12] showed that if $f \leqslant(1+o(1)) n / \ln n$, then Forcer wins $\mathcal{A} \mathcal{F}_{\text {mon }}\left(\mathcal{H a m}_{n}, 1, f\right)$ (and therefore wins also $\mathcal{A} \mathcal{F}_{\text {mon }}\left(\mathcal{M}_{n}, 1, f\right)$ ). The same condition on $f$ suffices to force Avoider to create a spanning $d$-connected subgraph of $K_{n}$, so $\mathcal{A} \mathcal{F}_{\text {mon }}\left(\mathcal{D}_{d, n}, 1, f\right)$ and $\mathcal{A} \mathcal{F}_{\text {mon }}\left(\mathcal{C}_{d, n}^{\prime}, 1, f\right)$ can be won by Forcer. On the other hand, Hefetz et al. [9] proved that if $f>(1+o(1)) n / \ln n$, then Avoider wins $\mathcal{A F}_{\text {mon }}\left(\mathcal{D}_{1, n}, 1, f\right)$. Hence we have

$$
f_{\mathcal{D}_{d, n}}^{\text {mon }}, f_{\mathcal{C}_{d, n}^{\prime}}^{\text {mon }}, f_{\mathcal{C}_{d, n}}^{\text {mon }}, f_{\mathcal{H}^{\text {mam }} n}^{\text {mon }}, f_{\mathcal{M}_{n}}^{\text {mon }}=(1+o(1)) \frac{n}{\ln n} .
$$

So the leading term of the threshold in all the above games is the same as in the corresponding Maker-Breaker games ([5],[7],[11]).

The strict versions of the above Avoider-Forcer games are harder to study and we know only partial results for some of these games. Hefetz, Krivelevich and Szabó [10] proved that for the connectivity game $\mathcal{A F}\left(\mathcal{C}_{1, n}, 1, f\right)$ the threshold exists and

$$
f_{\mathcal{C}_{1, n}}=\left\lfloor\frac{n-1}{2}\right\rfloor \text {. }
$$

For the games $\mathcal{A} \mathcal{F}\left(\mathcal{M}_{n}, 1, f\right)$ and $\mathcal{A} \mathcal{F}(\mathcal{H a m}, 1, f)$ the orders of the upper and the lower thresholds are not known. Hefetz, Krivelevich and Szabó [10] proved that $f_{\mathcal{M}_{n}}^{-}=$ $\Omega(n / \ln n)$, and due to Krivelevich and Szabó [12] we have $f_{\mathcal{H} a m_{n}}^{-}=\Omega(n / \ln n)$. As for the upper threshold, a simple calculation of the number of edges needed to create a perfect matching or a Hamilton cycle in $K_{n}$ implies that $f_{\mathcal{M}_{n}}^{+}<n$ and $f_{\mathcal{H} a m_{n}}^{+}<0.5 n$. Although in this paper we improve slightly these bounds (see Corollary 1.8 below), the questions whether $f_{\mathcal{M}_{n}}^{+}=o(n)$ and $f_{\mathcal{H} a m_{n}}^{+}=o(n)$ remain widely open.

Thresholds for small-graph Avoider-Forcer games, even in the monotone version, often differ from the thresholds of Maker-Breaker games. Hefetz et al. [9] showed that for the triangle game we have

$$
f_{\mathcal{H}_{K_{3}, n}}^{\text {mon }}=\Theta\left(n^{3 / 2}\right)
$$

while in the Maker-Breaker triangle game the threshold is of order $\sqrt{n}$ (cf. [5]). In general, for every graph $G$ with at least two edges, the Maker-Breaker threshold is of order $n^{1 / m_{2}(G)}$, 
where $m_{2}(G)=\max \{(e(F)-1) /(v(F)-2): F \subseteq G, e(F) \geqslant 2\}$, and $v(F)$ and $e(F)$ denote the number of vertices and edges of $F$ respectively (cf. [4]).

As for the strict version of small-graph games, the order of the upper or the lower threshold is not known for any graph containing a cycle. Not much is known about the thresholds for trees. For a path $P_{3}$ on three vertices we have $f_{\mathcal{H}_{P_{3}, n}}^{-}=\Theta\left(n^{3 / 2}\right)$ and $f_{\mathcal{H}_{P_{3}, n}}^{+}=\left(\begin{array}{l}n \\ 2\end{array}\right)-2([9])$. We should also mention that Ferber, Krivelevich and Naor [6] considered strict Avoider-Forcer $(a: f)$ small-graph games played on dense graphs with $n$ vertices. They found a condition sufficient for forcing Avoider to build a copy of a fixed graph $G$, provided $a$ and $f$ are fixed and $n$ is large. From their results we can infer that if $f+1$ is coprime to every number not greater than $e(G)$ and $f<c n^{2 / e(G)}$ with some constant $c$ depending on $G$, then Avoider in $\mathcal{A F}\left(\mathcal{H}_{G, n}, 1, f\right)$ is forced to build a copy of $G$ in $K_{n}$.

Here we will be primarily concerned with the upper and the lower thresholds in $d$-degree and small-graph games played on $K_{n}$. For 1-degree strict game, the result (2) implies that $f_{\mathcal{D}_{1, n}}^{-} \geqslant\lfloor(n-1) / 2\rfloor$. We will show that $\lfloor(n-1) / 2\rfloor$ is also the upper bound for $f_{\mathcal{D}_{1, n}}^{+}$and hence $f_{\mathcal{D}_{1, n}}^{-}=f_{\mathcal{D}_{1, n}}^{+}$; the only exceptions are $n=4$ and $n=7$.

Theorem 1.4. For every $n \geqslant 3$, if $n \neq 4,7$, then

$$
f_{\mathcal{D}_{1, n}}^{+} \leqslant\left\lfloor\frac{n-1}{2}\right\rfloor \text {. }
$$

One can easily check that $f_{\mathcal{D}_{1,4}}^{-}=1$ and $f_{\mathcal{D}_{1,4}}^{+}=4$. With some effort one can calculate that $f_{\mathcal{D}_{1,7}}^{-}=f_{\mathcal{D}_{1,7}}^{+}=4$.

Corollary 1.5. Let $n \geqslant 3$. If $n \neq 4$, then the threshold $f_{\mathcal{D}_{1, n}}$ exists, and for $n \neq 4,7$

$$
f_{\mathcal{D}_{1, n}}=f_{\mathcal{C}_{1, n}}=\left\lfloor\frac{n-1}{2}\right\rfloor
$$

For fixed $d \geqslant 2$ the inequality $f_{\mathcal{D}_{d, n}}^{+}<n / d$ is obvious, while the best known lower estimation of $f_{\mathcal{D}_{d, n}}^{-}$is $f_{\mathcal{D}_{d, n}}^{-} \geqslant n /(2 d)-1$, which is implied by the following result by Hefetz, Krivelevich and Szabó [10]:

$$
f_{\mathcal{C}_{d, n}^{\prime}}^{-} \geqslant \frac{n}{2 d}-1
$$

Therefore $n /(2 d)-1 \leqslant f_{\mathcal{D}_{d, n}}^{-} \leqslant f_{\mathcal{D}_{d, n}}^{+}<n / d$. It would be interesting to find the optimal constants $c, c^{\prime}>0$ (perhaps depending on $d$ ) such that $f_{\mathcal{D}_{d, n}}^{-} \geqslant c n / d$ and $f_{\mathcal{D}_{d, n}}^{+} \leqslant c^{\prime} n / d$. We did not succeed in finding such constants even for $d=2$, but we can prove that the upper bound $n / d$ on $f_{\mathcal{D}_{d, n}}^{+}$is not optimal even asymptotically.

Theorem 1.6. Let $d \geqslant 2$ be a fixed integer. Then

$$
f_{\mathcal{D}_{d, n}}^{+}<\frac{n}{\sqrt{(d-1)^{2}+1}+1}+o(n) .
$$


As far as strict $d$-degree games with $d=d(n) \rightarrow \infty$ are concerned, the upper and lower thresholds are close to $n / d$. It is a consequence of the obvious estimation $f_{\mathcal{D}_{d, n}}^{+}<n / d$ and Beck's results on discrepancy games, which we discuss in Section 3.

Theorem 1.7 (implicit in [3]). If $d=d(n)=\omega(\sqrt{n})$ then

$$
f_{\mathcal{D}_{d, n}}^{-}=(1+o(1)) \frac{n}{d} \quad \text { and } \quad f_{\mathcal{D}_{d, n}}^{+}=(1+o(1)) \frac{n}{d} .
$$

Theorems 1.4 and 1.6 imply some upper bounds on $f_{\mathcal{M}_{n}}^{+}$and $f_{\mathcal{H} a m_{n}}^{+}$. They are far from the known lower bounds $\Omega(n / \ln n)$, but are still a little bit better than the obvious estimations $f_{\mathcal{M}_{n}}^{+}<n$ and $f_{\mathcal{H} a m_{n}}^{+}<0.5 n$.

\section{Corollary 1.8.}

(i) $f_{\mathcal{M}_{n}}^{+} \leqslant\left\lfloor\frac{n-1}{2}\right\rfloor$ for every even $n \neq 4$.

(ii) $f_{\mathcal{H} a m_{n}}^{+}<0.42 n+o(n)$.

Finally let us mention some new results on small-graph games. To this end we define three graph parameters (here and below we assume that $e(G) \geqslant 2$ ):

$$
\begin{aligned}
m(G) & =\max _{F \subseteq G: v(F) \geqslant 1} \frac{e(F)}{v(F)}, \quad m^{\prime}(G)=\max _{F \subseteq G: v(F) \geqslant 1} \frac{e(F)-1}{v(F)}, \\
m_{2}(G) & =\max _{F \subseteq G: e(F) \geqslant 2} \frac{e(F)-1}{v(F)-2} .
\end{aligned}
$$

Based on Theorem 1.2, we will derive the following upper bounds on the monotone/upper/lower thresholds of $\mathcal{A} \mathcal{F}\left(\mathcal{H}_{G, n}, 1, f\right)$ and $\mathcal{A} \mathcal{F}_{\text {mon }}\left(\mathcal{H}_{G, n}, 1, f\right)$.

Theorem 1.9. Let $G$ be a graph with at least two edges. Then the following holds.

(i) $f_{\mathcal{H}_{G, n}}^{\text {mon }}=O\left(n^{1 / m^{\prime}(G)}\right)$ and $f_{\mathcal{H}_{G, n}}^{+}=O\left(n^{1 / m^{\prime}(G)}\right)$.

(ii) $f_{\mathcal{H}_{G, n}}^{-}=O\left(n^{1 / m(G)} \ln n\right)$.

(iii) $f_{\mathcal{H}_{G, n}}^{-}<c n^{1 / m(G)}$ for some constant $c$ and infinitely many $n$.

(iv) For some constant $c$, sufficiently large $n$, and $f>c n^{1 / m(G)}$, Avoider can avoid a copy of $G$ until the last but one turn of $\mathcal{A} \mathcal{F}\left(\mathcal{H}_{G, n}, 1, f\right)$ and $\mathcal{A} \mathcal{F}_{\text {mon }}\left(\mathcal{H}_{G, n}, 1, f\right)$.

Note that (i) gives an alternative proof of the fact that $f_{\mathcal{H}_{K_{3}, n}}^{\text {mon }}=O\left(n^{3 / 2}\right)$. In (ii) and (iii) the constant exponent $1 / m(G)$ cannot be improved in general, because for $G=P_{3}$ we have $f_{\mathcal{H}_{G, n}}^{-}=\Theta\left(n^{1 / m(G)}\right)$. For an application of (iv), consider the triangle monotone game $(1: f)$, with $f$ of order greater than $n$ but less than $n^{3 / 2}$. We know by (3) that Forcer has a winning strategy; (iv) implies that Avoider can defend almost to the very end of the game. 


\section{Theorem 1.10.}

(i) $f_{\mathcal{H}_{K_{3}, n}}^{-}=\Omega\left(n^{1 / 2}\right)$.

(ii) $f_{\mathcal{H}_{G, n}}^{-}=\Omega\left(n^{1 / m_{2}(G)} / \ln n\right)$ and $f_{\mathcal{H}_{G, n}}^{\text {mon }}=\Omega\left(n^{1 / m_{2}(G)} / \ln n\right)$ for every graph $G$ with at least two edges.

The first part of the above theorem is a consequence of a winning condition for Forcer, which is presented in the next section. The second part is implied by Theorem 1.1 and the recent hypergraph container theorem by Saxton and Thomason [13].

The paper is organized in the following way. In Section 2 we prove Theorems 1.2 and 1.3 and discuss some variants of Beck's winning condition for Forcer. In Section 3 we prove Theorems 1.4 and 1.6, and explain how Theorem 1.7 follows from Beck's results on discrepancy games. In Section 4 we prove Theorems 1.9 and 1.10.

\section{Winning criteria for Avoider and Forcer}

Apart from the definitions stated in the introduction, we will use the following notation.

By $\Delta(\mathcal{H})$ we denote the maximum degree of the hypergraph $\mathcal{H}$, i.e.

$$
\Delta(\mathcal{H})=\max _{x \in V(\mathcal{H})}|\{D \in E(\mathcal{H}): x \in D\}|
$$

Additionally, we set

$$
\Delta_{2}(\mathcal{H})=\max _{x, y \in V(\mathcal{H}), x \neq y}|\{D \in E(\mathcal{H}): x, y \in D\}|
$$

Given a hypergraph $\mathcal{H}$ and $X \subseteq V(\mathcal{H})$, we denote by $\mathcal{H} \backslash X$ the hypergraph with the vertex-set $V(\mathcal{H}) \backslash X$ and the edge-multiset $\{D \backslash X: D \in E(\mathcal{H})\}$. By $\mathcal{H}-X$ we mean the hypergraph with the vertex-set $V(\mathcal{H}) \backslash X$ and the edge-multiset $\{D \in E(\mathcal{H}): D \cap X=\emptyset\}$. We mean by $\mathcal{H}_{X}$ the subhypergraph of $\mathcal{H}$, induced by all edges $D \in E(\mathcal{H})$ such that $X \subseteq D$. For simplicity we write $\mathcal{H}_{x_{1}, x_{2}, \ldots, x_{t}}$ instead of $\mathcal{H}_{\left\{x_{1}, x_{2}, \ldots, x_{t}\right\}}$.

Suppose that at a given moment of a game played on a hypergraph $\mathcal{H}$, the set $X$ consists of all vertices Avoider has selected so far and $Y$ is the set of vertices which belong to Forcer. Then we call the hypergraph $(\mathcal{H} \backslash X)-Y$ the position at that moment of the game. The position after the $i$ th turn will be denoted by $\mathcal{H}(i)$, with the technical assumption that $\mathcal{H}(0)=\mathcal{H}$.

Given a hypergraph $\mathcal{H}$ and a real number $\mu>0$, we define the weight $T_{\mu}$ of the hypergraph $\mathcal{H}$ as follows:

$$
T_{\mu}(\mathcal{H})=\sum_{D \in E(\mathcal{H})} \mu^{-|D|}
$$

Notice that in the final position $\mathcal{H}^{\prime}$ of an Avoider-Forcer game we have either $E\left(\mathcal{H}^{\prime}\right)=\emptyset$ and $T_{\mu}\left(\mathcal{H}^{\prime}\right)=0$ or $\emptyset \in E\left(\mathcal{H}^{\prime}\right)$ and $T_{\mu}\left(\mathcal{H}^{\prime}\right) \geqslant 1$. Hence the final position is won by Forcer if and only if its weight $T_{\mu}$ is positive. 


\subsection{Winning condition for Avoider}

Proof of Theorem 1.2. The proof of part (i) relies on the standard technique of minimizing the weight of the position, used by Beck for proving the winning condition (1) for Breaker in Maker-Breaker games. For completeness, we repeat briefly this argument and point out where our proof is different from the proof of Theorem 1.1.

Consider the weight function $T=T_{\mu}$ with $\mu=\frac{f}{a r}+1$ and suppose that Avoider uses a min-weight strategy. More precisely, for every $i \geqslant 0$, in turn $i+1$ Avoider selects $a$ vertices $x_{1}, \ldots, x_{a}$ such that $x_{1}$ minimizes $T\left(\mathcal{H}(i)_{x}\right)$ over all $x \in V(\mathcal{H}(i))$ and for $j=1, \ldots, a-1$ the vertex $x_{j+1}$ minimizes $T\left(\left(\mathcal{H}(i) \backslash\left\{x_{1}, \ldots, x_{j}\right\}\right)_{x}\right)$ over all $x \in V\left(\mathcal{H}(i) \backslash\left\{x_{1}, \ldots, x_{j}\right\}\right)$. We will show that the weight $T$ of the position, evaluated after every Forcer's move, does not increase in the course of the game.

Suppose that the play has $t$ turns and let $0 \leqslant i \leqslant t-2$. Let $X=\left\{x_{1}, \ldots, x_{a}\right\}$ and $Y=\left\{y_{1}, \ldots, y_{b}\right\}$ with $b \geqslant f$ be the sets of all vertices selected in the $(i+1)$ st turn by Avoider and Forcer respectively. Notice that based on the definition of the min-weight strategy and the definition of $T$,

$$
T\left(\mathcal{H}(i)_{x_{1}}\right) \leqslant T\left(\mathcal{H}(i)_{x_{a}}\right) \leqslant T\left(\left(\mathcal{H}(i) \backslash\left\{x_{1}, \ldots, x_{a-1}\right\}\right)_{x_{a}}\right),
$$

and similarly, for $j=1, \ldots, a-2$,

$$
\begin{aligned}
T\left(\left(\mathcal{H}(i) \backslash\left\{x_{1}, \ldots, x_{j}\right\}\right)_{x_{j+1}}\right) & \leqslant T\left(\left(\mathcal{H}(i) \backslash\left\{x_{1}, \ldots, x_{j}\right\}\right)_{x_{a}}\right) \\
& \leqslant T\left(\left(\mathcal{H}(i) \backslash\left\{x_{1}, \ldots, x_{a-1}\right\}\right)_{x_{a}}\right) .
\end{aligned}
$$

Furthermore, the function $T$, evaluated after selecting the vertices by Avoider, equals

$$
T(\mathcal{H}(i))+(\mu-1)\left[T\left(\mathcal{H}(i)_{x_{1}}\right)+T\left(\left(\mathcal{H}(i) \backslash\left\{x_{1}\right\}\right)_{x_{2}}\right)+\ldots+T\left(\left(\mathcal{H}(i) \backslash\left\{x_{1}, \ldots, x_{a-1}\right\}\right)_{x_{a}}\right)\right] .
$$

Hence Avoider's move $X$ increases $T$ by not more than

$$
a(\mu-1) T\left(\left(\mathcal{H}(i) \backslash\left\{x_{1}, \ldots, x_{a-1}\right\}\right)_{x_{a}}\right) .
$$

Starting with $T\left(\mathcal{H}(i) \backslash\left\{x_{1}, \ldots, x_{a}\right\}\right)$, we will estimate the decrease of $T$ caused by Forcer's choice of $Y$. At this point our analysis veers away from the proof of Theorem 1.1.

After the move of Forcer, $T$ decreases by

$$
\begin{aligned}
\sum_{\substack{D \in E\left(\mathcal{H}(i) \backslash\left\{x_{1}, \ldots, x_{a}\right\}\right): \\
D \cap Y \neq \emptyset}} \mu^{-|D|} & \geqslant \frac{1}{r} \sum_{\substack{D \in E\left(\mathcal{H}(i) \backslash\left\{x_{1}, \ldots, x_{a}\right\}\right): \\
D \cap Y \neq \emptyset}}|D| \mu^{-|D|} \geqslant \frac{1}{r} \sum_{y \in Y} \sum_{\substack{D \in E\left(\mathcal{H}(i) \backslash\left\{x_{1}, \ldots, x_{a}\right\}\right): \\
y \in D}} \mu^{-|D|} \\
& =\frac{1}{r} \sum_{y \in Y} T\left(\left(\mathcal{H}(i) \backslash\left\{x_{1}, \ldots, x_{a}\right\}\right)_{y}\right) \\
& \geqslant \frac{1}{r} \sum_{y \in Y} T\left(\left(\mathcal{H}(i) \backslash\left\{x_{1}, \ldots, x_{a-1}\right\}\right)_{y}\right) \\
& \geqslant \frac{f}{r} T\left(\left(\mathcal{H}(i) \backslash\left\{x_{1}, \ldots, x_{a-1}\right\}\right)_{x_{a}}\right) .
\end{aligned}
$$


In the last inequality we used the property that $x_{a}$ minimizes $T\left(\left(\mathcal{H}(i) \backslash\left\{x_{1}, \ldots, x_{a-1}\right\}\right)_{x}\right)$ over all $x \in V\left(\mathcal{H}(i) \backslash\left\{x_{1}, \ldots, x_{a-1}\right\}\right)$.

Summarizing, the change of $T$ in the $(i+1)$ st turn is

$$
\begin{aligned}
T(\mathcal{H}(i+1))-T(\mathcal{H}(i)) \leqslant & a(\mu-1) T\left(\left(\mathcal{H}(i) \backslash\left\{x_{1}, \ldots, x_{a-1}\right\}\right)_{x_{a}}\right) \\
& -\frac{f}{r} T\left(\left(\mathcal{H}(i) \backslash\left\{x_{1}, \ldots, x_{a-1}\right\}\right)_{x_{a}}\right),
\end{aligned}
$$

which is nonpositive as $\mu=\frac{f}{a r}+1$. Consequently, we proved that $T(\mathcal{H}(i)) \leqslant T(\mathcal{H}(0))=$ $T(\mathcal{H})$ for $i=1, \ldots, t-1$.

Consider the last, $t$ th, turn; Avoider takes a set $X$ of $a$ elements (or less, if it is not possible) and Forcer takes some remaining elements. Then

$$
T(\mathcal{H}(t)) \leqslant \sum_{D \in E(\mathcal{H}(t-1))} \mu^{|D \cap X|} \mu^{-|D|} \leqslant \mu^{a} T(\mathcal{H}(t-1)) \leqslant \mu^{a} T(\mathcal{H})
$$

By the assumption of our theorem $T(\mathcal{H})<\mu^{-a}$, so the above inequality implies that $T(\mathcal{H}(t))<1$. It means that in the end position Avoider has no edge of $\mathcal{H}$ entirely occupied.

The proof of part (ii) is almost the same. It is even easier since we do not have to analyze the last turn separately.

Proof of Theorem 1.3. Suppose that $a, f, r, n \geqslant 1$ and $\mathcal{H}$ is a hypergraph with $n$ pairwise disjoint edges, each of size $r$. Ferber, Krivelevich and Naor [6] proved that if

$$
N(a, f, k)= \begin{cases}f+1 & \text { for } k<a+1, \\ f+1+\left\lceil\frac{f}{a+1}\right\rceil & \text { for } k=a+1, \\ \left\lceil\frac{N(a, f, k-1)}{a}\right\rceil\left(a+\left\lceil\frac{f}{k}\right\rceil\right) & \text { for } k>a+1,\end{cases}
$$

and $n \geqslant N(a, f, r)$, then Forcer wins $\mathcal{A F}_{\text {mon }}(\mathcal{H}, a, f)$.

Assume that $n=N(a, f, r), r \geqslant 3 a$, and $a, r$ can depend on $f$. Additionally, let $r a=o(f)$. We will show that

$$
n<\left(\frac{3 f}{a r}+1\right)^{r-a}, \quad \text { provided } f \text { is sufficiently large. }
$$

Clearly it will imply that $\mathcal{H}$ satisfies the assumption of Theorem 1.3 . It will also complete our proof because of the above mentioned result by Ferber, Krivelevich and Naor.

By the definition of $N(a, f, k)$, we have $N(a, f, k)>f$ and for $k>a+1$

$$
\begin{aligned}
N(a, f, k) & <\left(\frac{N(a, f, k-1)}{a}+1\right)\left(a+\frac{f}{k}+1\right) \\
& \leqslant N(a, f, k-1)\left(\frac{f}{a k}+2\right)+a+\frac{f}{k}+1 \\
& <N(a, f, k-1)\left(\frac{f}{a k}+2\right)+a+\frac{N(a, f, k-1)}{k}+1 \\
& <N(a, f, k-1)\left(\frac{f}{a k}+5\right),
\end{aligned}
$$


where in the last inequality we assumed that $a<f$. Hence, for $r a=o(f)$ we obtain

$$
\begin{aligned}
N(a, f, r) & <N(a, f, a+1)\left(\frac{f+o(f)}{a}\right)^{r-a-1} \prod_{k=a+2}^{r} \frac{1}{k} \\
& =\left(f+1+\left\lceil\frac{f}{a+1}\right]\right)\left(\frac{f+o(f)}{a}\right)^{r-a-1} \prod_{k=a+2}^{r} \frac{1}{k} \\
& =\frac{a+2}{a+1}(f+o(f))\left(\frac{f+o(f)}{a}\right)^{r-a-1} \prod_{k=a+2}^{r} \frac{1}{k} \\
& =\left(\frac{f+o(f)}{a}\right)^{r-a} a(a+2) \prod_{k=a+1}^{r} \frac{1}{k} \\
& =\left(\frac{f+o(f)}{a}\right)^{r-a} a(a+2) \frac{a !}{r !} .
\end{aligned}
$$

Thus for sufficiently large $f$

$$
\begin{aligned}
\frac{N(a, f, r)}{\left(\frac{3 f}{a r}+1\right)^{r-a}} & <(1+o(1))^{r-a} \frac{a ! a(a+2) r^{r-a}}{r ! 3^{r-a}}<\frac{a ! a(a+2) r^{r-a}}{r ! e^{r-a}} \\
& <\frac{a ! a(a+2) e^{a}}{\sqrt{2 \pi r} \cdot r^{a}}<\frac{a ! a(a+2)}{\sqrt{6 a \pi} \cdot a^{a}},
\end{aligned}
$$

where in the last inequality we used the assumption that $r \geqslant 3 a$. By routine calculations we check that the right-hand side of the obtained inequality is less than 1 for every $a \geqslant 1$.

Hence (5) holds and the proof of Theorem 1.3 is complete.

\subsection{Winning criteria for Forcer}

In this section we present a few winning criteria for Forcer in $\mathcal{A} \mathcal{F}(\mathcal{H}, a, f)$, which are slight modifications of Beck's winning condition from [3]. Let $\mathcal{F} \mathcal{A}(\mathcal{H}, a, f)$ denote the game which differs from $\mathcal{A} \mathcal{F}(\mathcal{H}, a, f)$ by only one rule, in $\mathcal{F} \mathcal{A}(\mathcal{H}, a, f)$ Forcer is the first player.

Theorem 2.1 (implicit in [3]). Suppose that

$$
T_{f / a+1}(\mathcal{H})=\sum_{D \in E(\mathcal{H})}\left(\frac{f}{a}+1\right)^{-|D|}>\frac{f^{2} a^{2}}{(f+a)^{3}} \Delta_{2}(\mathcal{H})|V(\mathcal{H})|
$$

and $f+a$ divides $|V(\mathcal{H})|$. Then Forcer has a winning strategy for $\mathcal{F} \mathcal{A}(\mathcal{H}, a, f)$.

Beck proved that (6) guarantees the win of Maker in $\mathcal{M B}(\mathcal{H}, a, f)$ (cf. [3], Theorem 2.2) and remarked that by a very similar proof, one can show the analogous result for Forcer in Avoider-Forcer games. It follows from the proof of Beck's Theorem 2.2 in [3] 
that the divisibility assumption in Theorem 2.1 is quite important, though irrelevant in the case of Maker-Breaker games.

We can formulate a theorem similar to Theorem 2.1 if Avoider is the first player, provided he takes the last unoccupied vertex in the game. Forcer can simply ignore the first move of Avoider, pretend he starts the game, and use a winning strategy guaranteed by Theorem 2.1 .

\section{Corollary 2.2. Suppose that}

$$
T_{f / a+1}(\mathcal{H})>\frac{f^{2} a^{2}}{(f+a)^{3}} \Delta_{2}(\mathcal{H})|V(\mathcal{H})|
$$

and $f+a$ divides $|V(\mathcal{H})|-a$. Then Forcer has a winning strategy for $\mathcal{A} \mathcal{F}(\mathcal{H}, a, f)$.

The technique of the proof of Theorem 2.1 allows us to solve the above divisibility problems in the following way.

Theorem 2.3. If

$$
T_{f / a+1}(\mathcal{H})>\frac{f^{2} a^{2}}{(f+a)^{3}} \Delta_{2}(\mathcal{H})|V(\mathcal{H})|+\Delta(\mathcal{H}) \frac{f a}{f+a},
$$

then Forcer has a winning strategy for $\mathcal{A} \mathcal{F}(\mathcal{H}, a, f), \mathcal{F} \mathcal{A}(\mathcal{H}, a, f)$ and $\mathcal{A F}_{\text {mon }}(\mathcal{H}, a, f)$.

Proof. Let $T=T_{f / a+1}$. The key realization for the proof of Theorem 2.1 (and the proof of Beck's Theorem 2.2 in [3]) is that Forcer in $\mathcal{F} \mathcal{A}(\mathcal{H}, a, f)$ can play so that for every $i$, if $|V(\mathcal{H}(i))| \geqslant a+f$, then

$$
T(\mathcal{H}(i+1)) \geqslant T(\mathcal{H}(i))-\left(\frac{f a}{f+a}\right)^{2} \Delta_{2}(\mathcal{H})
$$

Forcer in $\mathcal{A F}(\mathcal{H}, a, f)$ can use this strategy as well by ignoring the first move of Avoider. Furthermore, the same Forcer's strategy guarantees the property (7) in $\mathcal{A F}_{\text {mon }}(\mathcal{H}, a, f)$ since the assumption that Avoider can take more than $a$ vertices per turn is not relevant for the proof of Theorem 2.1.

Therefore, if $\mathcal{H}^{\prime}$ is the position before the last move of Forcer in $\mathcal{A F}(\mathcal{H}, a, f)$, or $\mathcal{F} \mathcal{A}(\mathcal{H}, a, f)$, or $\mathcal{A} \mathcal{F}_{\text {mon }}(\mathcal{H}, a, f)$, we have

$$
T\left(\mathcal{H}^{\prime}\right)>T(\mathcal{H})-\left(\frac{f a}{f+a}\right)^{2} \Delta_{2}(\mathcal{H}) \frac{|V(\mathcal{H})|}{f+a} .
$$

Consequently, by the assumption of Theorem 2.3 we obtain

$$
T\left(\mathcal{H}^{\prime}\right)>\Delta(\mathcal{H}) \frac{f a}{f+a} .
$$

Now we consider the effect of Forcer's last move. Every nonisolated vertex $x$ of $\mathcal{H}^{\prime}$ belongs to at most $\Delta(\mathcal{H})$ edges of size at least one, so $T\left(\mathcal{H}_{x}^{\prime}\right) \leqslant \Delta(\mathcal{H}) /(f / a+1)$. Forcer 
in his last move takes at most $f$ (remaining) vertices and hence the weight $T$ of the hypergraph obtained at the end of the game is not less than

$$
T\left(\mathcal{H}^{\prime}\right)-f \frac{\Delta(\mathcal{H})}{f / a+1}=T\left(\mathcal{H}^{\prime}\right)-\Delta(\mathcal{H}) \frac{f a}{f+a}
$$

which is positive.

We conclude that the end position is a win for Forcer.

\section{$3 \quad$ Degree games}

\subsection{Big degree games}

Let $b$ be a natural number. We have two players; Balancer who takes exactly $b$ free edges of $K_{n}$ per turn and Unbalancer who takes 1 free edge of $K_{n}$ per turn. Let $d_{B}(v)$ denote the number of Balancer's edges incident to $v \in V\left(K_{n}\right)$ at the end of the game. Balancer tries to get the degree balance roughly $b: 1$, i.e. he wants to obtain the number

$$
\max _{v \in V\left(K_{n}\right)}\left|d_{B}(v)-\frac{b(n-1)}{b+1}\right|
$$

as small as possible. We call this game the discrepancy $(b: 1)$ game on $K_{n}$. By default Balancer is the first player. However, we will also consider the discrepancy $(b: 1)$ games in which Unbalancer starts the game.

The discrepancy $(b: 1)$ game on $K_{n}$ belongs to a much more general class of discrepancy $(b: u)$ games on hypergraphs, with $u \geqslant 1$, studied by Beck [3]. Below we present a special case of Beck's result.

Theorem $3.1\left([3]\right.$, Thm. 27.5). Let $b=b(n)=o(n / \ln n)$ and $b+1 \mid\left(\begin{array}{l}n \\ 2\end{array}\right)$. Then for some constants $c, c^{\prime}>0$ Balancer as the first player has a strategy in the discrepancy $(b: 1)$ game on $K_{n}$ such that for every $x \in V\left(K_{n}\right)$

$$
\frac{b(n-1)}{b+1}-c^{\prime} \sqrt{\frac{n \ln n}{b+1}} \leqslant d_{B}(x) \leqslant \frac{b(n-1)}{b+1}+c \sqrt{\frac{n \ln n}{b+1}} .
$$

Remark. In the cited theorem of Beck there is no divisibility assumption, however, the method of the proof requires that every move of Balancer is followed by Unbalancer's move.

We can solve the problem of the last turn and get rid of the assumption $b+1 \mid\left(\begin{array}{l}n \\ 2\end{array}\right)$ by replacing the condition (8) by

$$
\frac{b(n-1)}{b+1}-c^{\prime} \sqrt{\frac{n \ln n}{b+1}}-b \leqslant d_{B}(x) \leqslant \frac{b(n-1)}{b+1}+c \sqrt{\frac{n \ln n}{b+1}}+b,
$$

since in the last move Balancer cannot change degrees in his graph more than by $b$. 
If we assume that Balancer in the discrepancy game is the second player, we can ignore the first move of Unbalancer and obtain almost the same condition (8) for Balancer's degrees, provided $b+1 \mid\left(\begin{array}{l}n \\ 2\end{array}\right)-1$. The forgotten move of Unbalancer can influence Balancer's degrees only by one, which is asymptotically irrelevant. Again we can get rid of the assumption $b+1 \mid\left(\begin{array}{l}n \\ 2\end{array}\right)-1$ if we change the degree bounds by $b$. Summarizing, we obtain the following corollary.

Corollary 3.2. Let $b=b(n)=o(n / \ln n)$. Then for some constants $c, c^{\prime}>0$ Balancer as the second player in the discrepancy $(b: 1)$ game on $K_{n}$ has a strategy such that for every $x \in V\left(K_{n}\right)$

$$
\frac{b(n-1)}{b+1}-c^{\prime} \sqrt{\frac{n \ln n}{b+1}}-b \leqslant d_{B}(x) \leqslant \frac{b(n-1)}{b+1}+c \sqrt{\frac{n \ln n}{b+1}}+b .
$$

We are ready to justify Theorem 1.7 , which says that for $d=d(n)=\omega(\sqrt{n})$

$$
f_{\mathcal{D}_{d, n}}^{-}=(1+o(1)) \frac{n}{d} \quad \text { and } \quad f_{\mathcal{D}_{d, n}}^{+}=(1+o(1)) \frac{n}{d} .
$$

Proof of Theorem 1.7. Let $d=d(n)=\omega(\sqrt{n})$. Since $f_{\mathcal{D}_{d, n}}^{-} \leqslant f_{\mathcal{D}_{d, n}}^{+}<n / d$, it is enough to prove that $f_{\mathcal{D}_{d, n}}^{-} \geqslant(1+o(1)) \frac{n}{d}$.

Assume that Forcer has a winning strategy for $\mathcal{A F}\left(\mathcal{D}_{d, n}, 1, f\right)$. Of course $f \leqslant f_{\mathcal{D}_{d, n}}^{+}<$ $n / d$. Additionally, by (4) we know that if $f \leqslant n /(2 d)-1$, then Forcer can force Avoider to build a $d$-edge-connected spanning graph and therefore he can win $\mathcal{A F}\left(\mathcal{D}_{d, n}, 1, f\right)$. Therefore we assume that $n /(2 d) \leqslant f<n / d$.

We have $f=\Theta(n / d)=o(\sqrt{n})=o(n / \ln n)$, so Forcer in $\mathcal{A F}\left(\mathcal{D}_{d, n}, 1, f\right)$ can use the strategy of Balancer described in Corollary 3.2 with $b=f$. This way at the end of the game $\mathcal{A F}\left(\mathcal{D}_{d, n}, 1, f\right)$ the maximum degree of Forcer's graph is not greater than $\frac{(n-1) f}{f+1}+c \sqrt{\frac{n \ln n}{f+1}}+f$ for some constant $c>0$. It means that the minimum degree of Avoider's final graph is not less than

$$
\frac{n-1}{f+1}-c \sqrt{\frac{n \ln n}{f+1}}-f>\frac{n-1}{f+1}-c \sqrt{2 d \ln n}-\frac{n}{d} .
$$

The latter expression is not less than $d$ for

$$
f \leqslant \frac{n-1}{d+c \sqrt{2 d \ln n}+n / d}-1
$$

so for $d=\omega(\sqrt{n})$ we obtain

$$
f \leqslant(1+o(1)) \frac{n}{d}
$$

Consequently, Avoider loses $\mathcal{A F}\left(\mathcal{D}_{d, n}, 1, f\right)$ for every such $f$, which means that $f_{\mathcal{D}_{d, n}}^{-} \leqslant$ $(1+o(1)) \frac{n}{d}$. 


\subsection{Proof of Theorem 1.4}

Let $n \geqslant 3$ and $f>\left\lfloor\frac{n-1}{2}\right\rfloor$. It is easy to calculate that at the end of the game $\mathcal{A F}\left(\mathcal{D}_{1, n}, 1, f\right)$ Avoider will have at most $n-2$ edges occupied. We will show that Avoider wins by building first a tree as big as possible and then by continuing the play almost arbitrarily.

Avoider starts with selecting any edge of $K_{n}$ and then, in every position in which Avoider's edges span a tree $T$, he selects any free edge from $E\left(V(T), V\left(K_{n}\right) \backslash V(T)\right)$, if possible; by $E(A, B)$ we denote the set of all edges $v v^{\prime}$ with $v \in A$ and $v^{\prime} \in B$.

If he is able to continue to build his tree until the end of the game, then clearly at least one vertex in Avoider's graph remains isolated. By Avoider's graph at a given moment of the game we mean a graph with the vertex-set $V\left(K_{n}\right)$ and with the edges selected by Avoider so far. Therefore we can assume that in some turn Avoider cannot increase his tree.

Let $k$, with $2 \leqslant k \leqslant n-2$, be the first turn in which Avoider cannot extend his tree $T$, i.e. $|V(T)|=k$ and all the edges from $E\left(V(T), V\left(K_{n}\right) \backslash V(T)\right)$ have been selected by Forcer. Then the number of edges Forcer has selected so far is

$$
(k-1) f \geqslant\left|E\left(V(T), V\left(K_{n}\right) \backslash V(T)\right)\right|=k(n-k)
$$

and hence $n-k \leqslant f-1$. We will consider two cases: $n-k>2$ and $n-k=2$.

Suppose that $n-k>2$. For $i \geqslant k$, in his $i$ th move Avoider selects arbitrarily a free edge from $E(V(T), V(T))$ as long as there is any. Let $t$ be the last turn in which such free edges exist (and $t \geqslant k-1)$. Then we have at most $\left(\begin{array}{c}n-k \\ 2\end{array}\right)$ free edges left and $n-k$ isolated vertices in Avoider's graph. By simple calculations one can check that if $3 \leqslant n-k \leqslant f-1$, then $\left(\begin{array}{c}n-k \\ 2\end{array}\right) \leqslant(f+1)\left\lfloor\frac{n-k-1}{2}\right\rfloor$. Hence after turn $t$ the game will last at most $\left\lfloor\frac{n-k-1}{2}\right\rfloor$ turns. We infer that Avoider will leave one of his $n-k$ isolated vertices untouched, no matter how he plays in the remaining turns. Thus Avoider wins the game.

Now consider the case $n-k=2$. Since $k \geqslant 2$, we have $n \geqslant 4$. We have assumed that after turn $k-1$ the game has not ended yet, which implies that $(k-1)(f+1)<\left(\begin{array}{l}n \\ 2\end{array}\right)$. Additionally, the game lasts no more than $n-2$ turns so the $k$ th turn is the last one. Let us recall that after $k-1$ turns there are two isolated vertices in Avoider's graph, say $v$ and $v^{\prime}$. If there is any free edge except of $v v^{\prime}$, Avoider in his last move selects it and wins the game. Suppose that $v v^{\prime}$ is the only free edge before the $k$ th move of Avoider. Then $(k-1)(f+1)=\left(\begin{array}{l}n \\ 2\end{array}\right)-1$. By solving this equation for $k=n-2 \geqslant 2$ we obtain that $f+1=(n+1)(n-2) /(2(n-3))$, so either $n=f=4$ or $n=7$ and $f=4$.

In view of the above analysis, we conclude that Avoider wins $\mathcal{A F}\left(\mathcal{D}_{1, n}, 1, f\right)$ for every $f>\left\lfloor\frac{n-1}{2}\right\rfloor$, provided $n \neq 4,7$. Hereby the proof is complete.

Remark. For $n=7$ the above argument implies that $f_{\mathcal{D}_{1, n}}^{+} \leqslant 4$. It appears that $\mathcal{A F}\left(\mathcal{D}_{1,7}, 1,4\right)$ is a win for Forcer. Forcer wins the game if he stands by the following rules.

1) As long as there are at least two isolated vertices in Avoider's graph:

- He prevents Avoider from building any cycle. 
- In every move, if possible, Forcer selects free edges $v v^{\prime}$ such that $v$ and $v^{\prime}$ have positive degree in Avoider's graph; if there are no more of these edges, he finishes his move by selecting edges with exactly one endpoint of positive degree in Avoider's graph.

2) At the moment the sixth vertex, say $v_{6}$, of positive degree appears in Avoider's graph and there is still one isolated vertex $v_{7}$ in Avoider's graph, Forcer continues to play arbitrarily, leaving $v_{6} v_{7}$ free as long as possible.

We skip the proof that such a strategy can be carried out by Forcer, but we will verify that it is a winning strategy for him. Note that there are five edges in Avoider's final graph, and it contains no cycle by Forcer's strategy. Therefore, it has at least six vertices of positive degree. Consider the moment the sixth vertex, say $v_{6}$, of positive degree appears in Avoider's graph. Then if no vertex is isolated in Avoider's graph, Forcer wins the game. Thus suppose there is still one isolated vertex $v_{7}$ in Avoider's graph. It follows from 1) that the edge $v_{6} v_{7}$ is free. Forcer can continue to play arbitrarily, leaving $v_{6} v_{7}$ free until Avoider selects a free edge incident to $v_{7}$ (notice that Avoider selects the last free edge in the game). Thereby Forcer wins $\mathcal{A} \mathcal{F}\left(\mathcal{D}_{1,7}, 1,4\right)$.

\subsection{Proof of Theorem 1.6}

Throughout this section we assume that $d \geqslant 2$. Additionally, we consider $f \geqslant n /(2 d)$ only, because otherwise, in view of (4), Forcer wins $\mathcal{A F}\left(\mathcal{D}_{d, n}, 1, f\right)$. Another assumption is that $f+1 \leqslant(n-1) / d$; otherwise the game $\mathcal{A F}\left(\mathcal{D}_{d, n}, 1, f\right)$ lasts less than $n d / 2$ turns and Avoider trivially wins.

The idea of the proof is quite simple. We will show that Avoider can build a spanning tree with many leaves. If $f$ is greater than, roughly, $\frac{n}{\sqrt{(d-1)^{2}+1}+1}$, there will be too few moves left for Avoider to increase the degree of every leaf by $d-1$.

Let us assume that Forcer has a winning strategy for $\mathcal{A} \mathcal{F}\left(\mathcal{D}_{d, n}, 1, f\right)$. In the lemma below by a greedy algorithm we mean any strategy of Avoider such that after every his move the graph induced by Avoider's edges is a tree.

Lemma 3.3. If Forcer has a winning strategy for $\mathcal{A F}\left(\mathcal{D}_{d, n}, 1, f\right)$, then Avoider can build a spanning tree within the first $n-1$ turns of the game by using any greedy algorithm.

Proof. Suppose for contradiction that Avoider builds greedily his tree, but after $k-1<$ $n-1$ turns he got stuck. Then $n-k \leqslant f-1$, as we already calculated in the proof of Theorem 1.4. Avoider can win the game continuing the play in the following way. Let $T$ be his tree built within $k-1$ first moves. Avoider takes free edges with both endpoints in $V(T)$ as long as possible. Then he forgets about the set $V(T)$ and transforms the game into $\mathcal{A F}\left(\mathcal{D}_{d, n-k}, 1, f\right)$, played on the complete graph $K_{n-k}$ with the vertex-set $V\left(K_{n}\right) \backslash V(T)$, possibly with some edges of $K_{n-k}$ occupied already by Forcer. Such a game, for $d \geqslant 2$ and $n-k \leqslant f-1$, has less than $(n-k) d / 2$ turns. Therefore at the end of the game at least one vertex of $K_{n-k}$ will have degree less than $d$ in Avoider's graph, which contradicts the assumption that Forcer wins. 
Now we describe how Avoider builds a rooted spanning tree in $\mathcal{A} \mathcal{F}\left(\mathcal{D}_{d, n}, 1, f\right)$. It is a greedy, BFS-like algorithm. Starting with any vertex $v_{0}$, the root, in consecutive turns $j \geqslant 1$ Avoider increases his tree on the vertex-set $\left\{v_{0}, v_{1}, \ldots, v_{j-1}\right\}$ by a vertex $v_{j}$ in the following way. Avoider finds the smallest $i \leqslant j-1$ such that for some $x \notin\left\{v_{0}, v_{1}, \ldots, v_{j-1}\right\}$ the edge $v_{i} x$ is free. Then he selects arbitrarily one such free edge and denotes its end (other than $v_{i}$ ) by $v_{j}$. We know by Lemma 3.3 that Avoider can continue building his tree for $j=1, \ldots, n-1$.

Consider the situation after the $(n-1)$ st turn. Let $T$ be the spanning tree of Avoider and $L$ be the set of its leaves. Throughout the rest of the proof the terms: leaves, parents, children, internal vertices, refer always to $T$. By the level of a vertex of $T$ we mean the distance in $T$ between $x$ and $v_{0}$. We say that $v_{i}$ is younger than $v_{j}$ if $i>j$. By $L_{k}$ we denote the set of $k$ youngest leaves of $T$, for $k=1, \ldots,|L|$. Avoider's degree of a vertex $x$ at a given moment of the game is the number of edges incident to $x$, selected by Avoider so far.

The following two lemmata will allow us to prove that $T$ has many leaves.

Lemma 3.4. Let $k \geqslant 3$. Suppose that $\left(\begin{array}{c}k \\ 2\end{array}\right) \leqslant f+1$ and there are $k$ vertices in $L$ with at most $t$ free edges of $K_{n}$ incident to each of them. If

$$
t \leqslant\left(d-1-\frac{2}{k}\right)(f+1)
$$

then Avoider has a strategy such that at the end of $\mathcal{A} \mathcal{F}\left(\mathcal{D}_{d, n}, 1, f\right)$ there is a vertex of degree less than d in Avoider's graph.

Proof. Let $L^{\prime} \subseteq L$ be a set of $k$ vertices satisfying the assumptions of the lemma. Avoider continues the game in the following way. In the first stage, as long as possible, he selects free edges not incident to any vertex from $L^{\prime}$. After the first stage there are at most $t k$ free edges of $K_{n}$ left. In the second stage Avoider selects free edges from $E\left(L^{\prime}, V\left(K_{n}\right) \backslash L^{\prime}\right)$, as long as there are any. Note that every such move of Avoider increases the degree of only one vertex in $L^{\prime}$ and after the second stage there are at most $\left(\begin{array}{l}k \\ 2\end{array}\right) \leqslant f+1$ free edges left. Thus we have at most one turn left after the second stage. In that turn Avoider increases by one the degree of two vertices of $L^{\prime}$.

Summarizing, after the first stage the game lasts no longer than $\lceil t k /(f+1)\rceil$ turns and the sum of Avoider's degrees of vertices in $L^{\prime}$ increases at most by $\lceil t k /(f+1)\rceil+1$. Therefore, if $t k \leqslant((d-1) k-2)(f+1)$, then at least one vertex from $L^{\prime}$ has degree less than $d$ at the end of $\mathcal{A F}\left(\mathcal{D}_{d, n}, 1, f\right)$.

Lemma 3.5. Let $3 \leqslant k \leqslant|L|$ and $x \in L_{k}$. Then there are less than $3 k$ internal vertices $y$ such that the edge $x y$ is free.

Proof. Let us start with the following observation, which is a result of the strategy of Avoider. We omit its straightforward inductive proof.

Observation. Suppose that $x, y \in V(T)$ and $y \notin L$. If an edge $x y$ is free, then $y$ is younger than the parent of $x$ and the level of $y$ differs from the level of $x$ by at most one. 
Suppose now that $x \in L_{k}$ and $l$ is the level of $x$. Since there are less than $k$ leaves younger than $x$, at level $l-1$ we have less than $k$ internal vertices younger than the parent of $x$. Also there are less than $k$ internal vertices at level $l$, and at level $l+1$ too. Thus, by the above Observation, there are less than $3 k$ internal vertices $y$ such that the edge $x y$ is free.

Observe that $|L| \geqslant d+1 \geqslant 3$. This fact is obvious if $d=3$ because of the greedy strategy of Avoider and the assumption that $d(f+1) \leqslant n-1$. Also in the case of $d=2$ Avoider's tree has at least three leaves; from the greedy strategy of Avoider we infer that if he has selected only two edges incident to the root $v_{0}$, then the child $v_{1}$ has degree greater than two in Avoider's graph. Therefore we can apply Lemmata 3.4 and 3.5 with $k=3$.

By Lemma 3.4 and the assumption that Avoider cannot win the game, we obtain that for some $x \in L_{3}$ the number of all free edges incident to $x$ is greater than $\left(d-1-\frac{2}{3}\right)(f+1)=$ $\left(d-\frac{5}{3}\right)(f+1)$. Furthermore, by Lemma 3.5, less than nine free edges join $x$ with internal vertices. Consequently

$$
|L|>\left(d-\frac{5}{3}\right)(f+1)-9>5 \sqrt{f}
$$

for sufficiently big $f$ or, equivalently, for big $n$ (we assumed that $n /(2 d) \leqslant f<n / d$ ).

In view of the above inequality we can apply Lemmata 3.4 and 3.5 again, with $k=$ $\lceil\sqrt{f}\rceil$. Hence for some $x \in L_{k}$ there are more than $\left(d-1-\frac{2}{k}\right)(f+1)-3 k>(d-1) f-5 \sqrt{f}$ leaves adjacent to $x$ with a free edge. We conclude that

$$
|L|>(d-1) f-5 \sqrt{f} .
$$

Let us recall that so far we have analyzed $(n-1)$ turns, so in the game there are $\left\lceil\left(\begin{array}{l}n \\ 2\end{array}\right) /(f+1)\right\rceil-(n-1)$ turns left. We also assumed that Forcer has a winning strategy. Hence Avoider's degree of every vertex in $L$ has to increase during these remaining turns from 1 up to $d$, which requires at least $|L|(d-1) / 2$ moves. Therefore

$$
\left\lceil\frac{\left(\begin{array}{l}
n \\
2
\end{array}\right)}{f+1}\right\rceil-(n-1) \geqslant \frac{1}{2}|L|(d-1)>\frac{1}{2}(d-1)^{2} f-\frac{5}{2}(d-1) \sqrt{f},
$$

then we obtain

$$
f<\frac{n}{\sqrt{(d-1)^{2}+1}+1}+o(n)
$$

which proves that $f_{\mathcal{D}_{d, n}}^{+}<\frac{n}{\sqrt{(d-1)^{2}+1}+1}+o(n)$.

\section{Small-graph games}

In strict Avoider-Forcer games on $K_{n}$ the number of free vertices before the last turn usually plays an important role. We need the following number theory facts.

Fact 4.1. Let $r<2$ be a rational number and $c>0$ be an integer. 
(i) There are infinitely many natural numbers $n$ such that $q \mid\left(\begin{array}{l}n \\ 2\end{array}\right)$ for some $q$ with $c n^{r}<q<2 c n^{r}$.

(ii) There are infinitely many natural numbers $n$ such that $q \mid\left(\begin{array}{l}n \\ 2\end{array}\right)-1$ for some $q$ with $c n^{r}<q<4 c n^{r}$.

Proof. Let us fix natural numbers $c, r_{1}, r_{2}$ and let $r=r_{1} / r_{2}<2$.

In order to prove (i), for every integer $k \geqslant 2$ we put $n-1=(4 c)^{k r_{2}}$ and define $q=2 c(n-1)^{r}=2 c(4 c)^{k r_{1}}$ if $r<1$, or $q=2 c(n-1)^{r-1} n=2 c(4 c)^{k\left(r_{1}-r_{2}\right)} n$ if $r \geqslant 1$. Then we obtain $c n^{r}<q<2 c n^{r}$ and $q \mid\left(\begin{array}{l}n \\ 2\end{array}\right)$. Thus we have infinitely many $n$ satisfying (i).

To show (ii), for every integer $k \geqslant 2$ we put $n-2=(4 c)^{k r_{2}}$ and define $q=2 c(n-2)^{r}$ if $r<1$, or $q=2 c(n-2)^{r-1}(n+1)$ if $r \geqslant 1$. By simple calculations one can verify that $c n^{r}<q<4 c n^{r}$, and $q \mid\left(\begin{array}{l}n \\ 2\end{array}\right)-1$ since $\left(\begin{array}{l}n \\ 2\end{array}\right)-1=(n+1)(n-2) / 2$. This proves (ii).

Fact 4.2. Let $\delta \in(0,1)$ and $N, q>0$ be integers such that $N^{\delta}<q<\delta N /(2 \ln N)$. Then, if $N$ is sufficiently large, there exists an integer $k$ such that $q \leqslant k \leqslant 2 \delta^{-1} q \ln q$ and the remainder of the division of $N$ by $k$ is greater than $q$.

Proof. Suppose that for every integer $k$ from the interval $\left[q, 2 \delta^{-1} q \ln q\right]$ the remainder of the division of $N$ by $k$ is at most $q$. In the interval $\left[q, 2 \delta^{-1} q \ln q\right]$ there are more than $(q+1) / \delta$ primes (for big $N)$, so more than $1 / \delta$ of them are equivalent modulo $N$. It means that for some $r \leqslant q, t>1 / \delta$ and some primes $p_{1}, \ldots, p_{t} \geqslant q$, we have $p_{i} \mid N-r$ for $i=1, \ldots, t$. Hence $p_{1} \cdot \ldots \cdot p_{t} \mid N-r$ and we conclude that $N>q^{t}>q^{1 / \delta}$. Therefore, if $q>N^{\delta}$, we come to a contradiction.

\subsection{Proof of Theorem 1.9}

Let us begin by a trivial observation. If Avoider can avoid building a subgraph of $G$ in $K_{n}$, then he avoids building the graph $G$ as well. Therefore, without loss of generality, while proving (i) we can assume that the maximum $m^{\prime}(G)$ is attained by $G$. Furthermore, (ii)(iv) obviously hold if $G$ is a matching (with at least two edges) so while proving (ii)-(iv) we will assume that $G$ contains two adjacent edges, and the maximum $m(G)$ is attained by $G$.

In order to prove (i), observe that there are $O\left(n^{v(G)}\right)$ copies of $G$ in $K_{n}$. Hence there exists a constant $c>0$ (which depends on $G$ ) such that if $f=\left\lceil\mathrm{cn}^{1 / \mathrm{m}^{\prime}(G)}\right\rceil=$ $\left\lceil c n^{v(G) /(e(G)-1)}\right\rceil$, then

$$
\sum_{A \in E\left(\mathcal{H}_{G, n}\right)}\left(\frac{f}{e(G)}+1\right)^{-e(G)+1}<1 .
$$

We have $\operatorname{rank}\left(\mathcal{H}_{G, n}\right)=e(G)$ so Avoider has a winning strategy for both $\mathcal{A F}\left(\mathcal{H}_{G, n}, 1, f\right)$ and $\mathcal{A F}_{\text {mon }}\left(\mathcal{H}_{G, n}, 1, f\right)$, based on the first part of Theorem 1.2. This proves that $f_{\mathcal{H}_{G, n}}^{\text {mon }}=$ $O\left(n^{1 / m^{\prime}(G)}\right)$ and $f_{\mathcal{H}_{G, n}}^{+}=O\left(n^{1 / m^{\prime}(G)}\right)$. 
To prove (ii), similarly observe that there is a constant $c \geqslant 1$ such that if $f=$ $\left\lceil c n^{1 / m(G)}\right\rceil=\left\lceil c n^{v(G) / e(G)}\right\rceil$, then

$$
\sum_{A \in E\left(\mathcal{H}_{G, n}\right)}\left(\frac{f}{e(G)}+1\right)^{-e(G)}<1 .
$$

Note that $m(G)>1 / 2$ for $G$ containing two adjacent edges, and the assumptions of Fact 4.2 are satisfied for $\delta=1 /(2 m(G)), N=\left(\begin{array}{l}n \\ 2\end{array}\right)$ and $q=f$, provided $n$ is sufficiently large. Thus there exists an integer $f^{\prime}$ such that $f \leqslant f^{\prime}+1 \leqslant 4 m(G) f \ln f$ and the remainder of a division of $\left(\begin{array}{l}n \\ 2\end{array}\right)$ by $f^{\prime}+1$ is greater than $f$. Hence, in the course of the game $\mathcal{A F}\left(\mathcal{H}_{G, n}, a, f^{\prime}\right)$, before every move of Avoider there are at least $f+1$ free vertices of the hypergraph. By the second part of Theorem 1.2, we conclude that Avoider will never occupy any edge entirely. It means that $f_{\mathcal{H}_{G, n}}^{-}<f^{\prime}=O\left(n^{1 / m(G)} \ln n\right)$.

The proof of part (iii) is similar to the previous one. We find an integer constant $c>0$

such that if $f \geqslant c n^{1 / m(G)}-1$, then $\sum_{A \in E\left(\mathcal{H}_{G, n}\right)}\left(\frac{f}{e(G)}+1\right)^{-e(G)}<1$. Based on the first part of Fact 4.1, we can argue that there are infinitely many natural numbers $n$ such that for some $f$ with $c n^{1 / m(G)}<f+1<2 c n^{1 / m(G)}$ we have $f+1 \mid\left(\begin{array}{l}n \\ 2\end{array}\right)$. For every such $n$ and $f$, in view of the second part of Theorem 1.2, Avoider wins $\mathcal{A} \mathcal{F}\left(\mathcal{H}_{G, n}, 1, f\right)$. Therefore $f_{\mathcal{H}_{G, n}}^{-}<2 c n^{1 / m(G)}$.

Finally, part (iv) is an immediate consequence of the second part of Theorem 1.2.

\subsection{Proof of Theorem $\mathbf{1 . 1 0}$}

To prove part (i), observe that for some constant $c$ and every sufficiently large $n$, if $f<c n^{1 / 2}$, then the inequality in Theorem 2.3 holds with $a=1$ and $\mathcal{H}=\mathcal{H}_{K_{3}, n}$. This is why Forcer in $\mathcal{A F}\left(\mathcal{H}_{K_{3}, n}, 1, f\right)$ has a winning strategy.

Now we will prove part (ii). Let $G$ be a graph with at least two edges. The key ingredient of the proof is a theorem by Saxton and Thomason [13] about $H$-free hypergraphs, which is a consequence of their much more general result on the so-called hypergraph containers. We should mention that another version of the hypergraph container theorem was independently proved by Balogh, Morris and Samotij [1]. Below we present the theorem by Saxton and Thomason in a simplified form, for $G$-free graphs.

Theorem 4.3 ([13], Thm 1.3). Let $G$ be a graph with $e(G) \geqslant 2$ and let $\varepsilon>0$. For some constant $c>0$ and sufficiently large $n$ there exists a collection $\mathcal{C}$ of subgraphs of $K_{n}$ such that

(a) for every $G$-free subgraph $F$ of $K_{n}$ there exists $C \in \mathcal{C}$ with $F \subseteq C$,

(b) $e(C) \leqslant(1-1 /(\chi(G)-1)+\varepsilon)\left(\begin{array}{c}n \\ 2\end{array}\right)$ for every $C \in \mathcal{C}$,

(c) $\ln |\mathcal{C}| \leqslant c n^{2-1 / m_{2}(G)} \ln n$. 
Let a family $\mathcal{C}$ and a constant $c>0$ be as described in the above theorem for $\varepsilon<1 /(\chi(G)-1)$. Observe that if the end position of $\mathcal{A F}\left(\mathcal{H}_{G, n}, 1, f\right)$ or $\mathcal{A F}_{\text {mon }}\left(\mathcal{H}_{G, n}, 1, f\right)$ is a win for Avoider, then Avoider's graph is contained in some $C \in \mathcal{C}$. Equivalently, Forcer has selected all edges in the set $E\left(K_{n}\right) \backslash E(C)$. Hence, Forcer has a winning strategy for $\mathcal{A F}\left(\mathcal{H}_{G, n}, 1, f\right)$ (or $\mathcal{A} \mathcal{F}_{\text {mon }}\left(\mathcal{H}_{G, n}, 1, f\right)$ ) if he can win playing Avoider's role in $\mathcal{A F}(\mathcal{H}, f, 1)$ $\left(\right.$ or $\mathcal{A F}_{\text {mon }}(\mathcal{H}, f, 1)$ ), where $V(\mathcal{H})=E\left(K_{n}\right)$ and $E(\mathcal{H})=\left\{E\left(K_{n}\right) \backslash E(C): C \in \mathcal{C}\right\}$.

Let $\delta>0$ be a constant such that $(1 /(\chi(G)-1)-\varepsilon)\left(\begin{array}{l}n \\ 2\end{array}\right)>\delta n^{2}$. By Theorem 4.3 we have

$$
\ln |E(\mathcal{H})| \leqslant c n^{2-1 / m_{2}(G)} \ln n \text { and }|D| \geqslant\left(\frac{1}{\chi(G)-1}-\varepsilon\right)\left(\begin{array}{l}
n \\
2
\end{array}\right)>\delta n^{2}
$$

for every $D \in E(\mathcal{H})$. Thus

$$
\sum_{D \in E(\mathcal{H})}\left(\frac{1}{f}+1\right)^{-|D|+f}<|E(\mathcal{H})|\left(\frac{1}{f}+1\right)^{-\delta n^{2}+f}<e^{c n^{2-1 / m_{2}(G)} \ln n} \cdot 2^{-\delta n^{2} / f+1},
$$

provided that $\delta n^{2}>f$. Calculations show that the right-hand side of the above inequality is less than 1 if $f<c^{\prime} n^{1 / m_{2}(G)} / \ln n$, where $c^{\prime}>0$ is some constant and $n$ is sufficiently large.

Therefore, for $f<c^{\prime} n^{1 / m_{2}(G)} / \ln n$, the assumption of the first part of Theorem 1.1 is satisfied (with $a=f$ ), so Avoider in $\mathcal{A F}(\mathcal{H}, f, 1)$ and $\mathcal{A F}_{\text {mon }}(\mathcal{H}, f, 1)$ has a winning strategy. It means that Forcer has a winning strategy for $\mathcal{A F}\left(\mathcal{H}_{G, n}, 1, f\right)$ and $\mathcal{A F}_{\text {mon }}\left(\mathcal{H}_{G, n}, 1, f\right)$, which completes the proof of Theorem 1.10.

\section{Acknowledgements}

I would like to thank the referees for their very thorough comments, which greatly improved the paper.

\section{References}

[1] J. Balogh, R. Morris, and W. Samotij. Independent sets in hypergraphs. preprint, arXiv: 1204.6530.

[2] J. Beck. Remarks on positional games. I. Acta Math. Acad. Sci. Hungar., 40:65-71, 1982.

[3] J. Beck. Combinatorial games: Tic-tac-toe theory. Encyclopedia of Mathematics and its Applications, no. 114, Cambridge University Press, Cambridge, 2008. xiv+732 pp.

[4] M. Bednarska and T. Łuczak. Biased positional games for which random strategies are nearly optimal. Combinatorica, 20:477-488, 2000.

[5] V. Chvátal and P. Erdős. Biased positional games. Annals of Discrete Math., 2:221228, 1978.

[6] A. Ferber, M. Krivelevich, and A. Naor. Avoider-Enforcer games played on edge disjoint hypergraphs. Dicrete Math., 313:2932-2941, 2013. 
[7] H. Gebauer and T. Szabó. Asymptotic random graph intuition for the biased connectivity game. Random Struct. Algor., 35:431-443, 2009.

[8] D. Hefetz, M. Krivelevich, M. Stojaković, and T. Szabó. Planarity, colorability and minor games. SIAM Journal on Discrete Mathematics, 22:194-212, 2008.

[9] D. Hefetz, M. Krivelevich, M. Stojaković, and T. Szabó. Avoider-Enforcer: The rules of the game. J. Combinatorial Theory Ser. A, 117:152-163, 2010.

[10] D. Hefetz, M. Krivelevich, and T. Szabó. Avoider-Enforcer games. J. Combinatorial Theory Ser. A, 114:840-853, 2007.

[11] M. Krivelevich. The critical bias for the Hamiltonicity game is $(1+\mathrm{o}(1)) \mathrm{n} / \mathrm{ln} \mathrm{n}$. Journal of the American Mathematical Society, 24:125-131, 2011.

[12] M. Krivelevich and T. Szabó. Biased positional games and small hypergraphs with large covers. Electronic Journal of Combinatorics, 15:publ. R70, 2008.

[13] D. Saxton and A. Thomason. Hypergraph containers. preprint, arXiv:1204.6595. 